\title{
Charge control of second life EV batteries on the DC link of a back-to-back converter
}

\author{
Myriam Neaimeh ${ }^{凶}$, Neal Wade, Simon Blake, Phil Taylor \\ Newcastle University, Newcastle upon Tyne, UK \\ $\bowtie$ E-mail: myriam.neaimeh@ncl.ac.uk
}

\begin{abstract}
Rural electricity customers are often subject to weak single-phase network connections. In this circumstance, powering three-phase equipment is not straightforward. This study addresses this problem through the development of a single- to three-phase power conversion system which is supplemented by the addition of energy storage on the DC bus of the converter. The storage is provided by a pair of second life electric vehicle (EV) batteries and charging is controlled by adjusting the DC bus voltage. The approach to, and challenges encountered in, developing this system are presented.
\end{abstract}

\section{Introduction}

One of the issues faced by rural electricity customers in the UK is weak single-phase supplies that are quite distant from the nearest substation. Providing single-phase power is less costly than providing a third conductor but could result in power capacity constraints and prohibit the installation of new sustainable technologies $[1,2]$.

Installing sustainable technologies could help rural communities and the UK as a whole achieve secure, economic and low carbon energy production and consumption. Anaerobic digesters (AD) are an example of sustainable technologies that can be installed on farms. An $\mathrm{AD}$ unit takes waste from the farm and turns it into biogas, digestate and water. The digestate is used as soil conditioner, and fertiliser, while the biogas can be used for transport, heating, electricity, or combined heat and power (CHP) applications, meeting some of the farm's energy needs [3].

While installing an $\mathrm{AD}$ unit presents farms with several advantages, there are issues that could prohibit the installation of such equipment. One of the issues is that such equipment with a large motor would require a non-existent three-phase connection. To avoid costly and disruptive traditional reinforcement such as building additional circuits to provide a three-phase connection, an alternative reinforcement approach is to use smart grid technologies such as a back-to-back converter. A back-to-back converter will provide the required three-phase power to the AD plant by power electronic conversion. The converter includes a rectifier which converts the utility supply to an intermediate DC link and a three-phase inverter that provides an AC waveform of the desired frequency and voltage level, see Fig. 1.

Another issue prohibiting the installation of AD units is that their full operation may exceed the capacity of the available grid supply of the farms. To avoid traditional reinforcement, an alternative would be to install energy storage. Energy storage, such as second life electric vehicle $(\mathrm{EV})$ batteries, can be used to strengthen the existing connection by accommodating a new load (e.g. large AD equipment).

The cost of Li-ion battery packs is decreasing continuously. This suggests that the cost of EVs will continue to decline and more users could be encouraged to adopt an electric car [4]. Repurposed automotive batteries can last several years in second use service if managed properly [5] and the capacity of a battery pack is over $80 \%$ of the original battery pack at the time of removal from the vehicle [6]. As such, identifying storage solutions using second life batteries will leverage on a potentially significant energy source.
This paper reports on the conceptual and practical issues that have arisen from a real-world demonstration on a farm that couples second life EV batteries to the DC link of a single-to three-phase back-to-back converter. This application combines the single- to three-phase converter and energy storage element in a cost effective, novel configuration.

\section{Application}

This work is based on the findings of the steps towards a real-world trial, at Newcastle University's Cockle Park farm, involving the installation of a $100 \mathrm{kVA}$ back-to-back converter to provide three-phase $A C$ supply to an $A D$ unit from the single-phase $A C$ grid supply, see Fig. 2. The biogas produced by the AD plant will be fed into a CHP plant which will be installed on the farm. The bespoke $100 \mathrm{kVA}$ converter includes the capacity for bi-directional power flow to enable the export of the electricity generated by the CHP back to the grid. In addition, access to the DC busbar of the converter is possible to allow battery storage and/or a Photovoltaic (PV) system to be connected to it directly.

The focus of this work is on the two used Nissan LEAF batteries (each battery capacity of $24 \mathrm{kWh}$, Fig. 3) that are installed in series on the DC-link busbar. Two batteries are needed for series connection to give a nominal $720 \mathrm{~V}$, matching the converter DC-link voltage. For the farm itself, one benefit of the batteries is that more of the energy generated by the future CHP can be used on the farm (at a different time), rather than exported at a lower price. For other farms and rural small and medium enterprises, the batteries are able to demonstrate other possibilities. In particular, similar batteries would allow equipment such as large motors to be installed and operated, even though their load may exceed the capacity of the available grid supply. In this trial, postulating a peak load of $80 \mathrm{~kW}$, for a duration of up to $5 \mathrm{~min} / \mathrm{h}$ (duration and frequency of the peak load of the anaerobic digester), in a farm with a connection limited to $50 \mathrm{~kW}$, the discrepancy can easily be made up by using the $48 \mathrm{kWh}$ capacity of the batteries.

Peak shaving would be achieved by connecting the system on a charge-discharge cycle of up to once per hour (e.g. discharging at up to $50 \mathrm{~kW}$, charging at up to $5 \mathrm{~kW}$ ). The $48 \mathrm{kWh}$ total (nominal) would allow for several discharge cycles before recharging. Each discharge up to $50 \mathrm{~kW}$ for $5 \mathrm{~min}=4 \mathrm{kWh}$.

As the batteries are from an EV, their battery management system is interfaced through a controller area network (CAN) controller. To safely operate the batteries a CAN interface had to be built to check safety parameters and monitor the state-of-charge, voltage and current. 


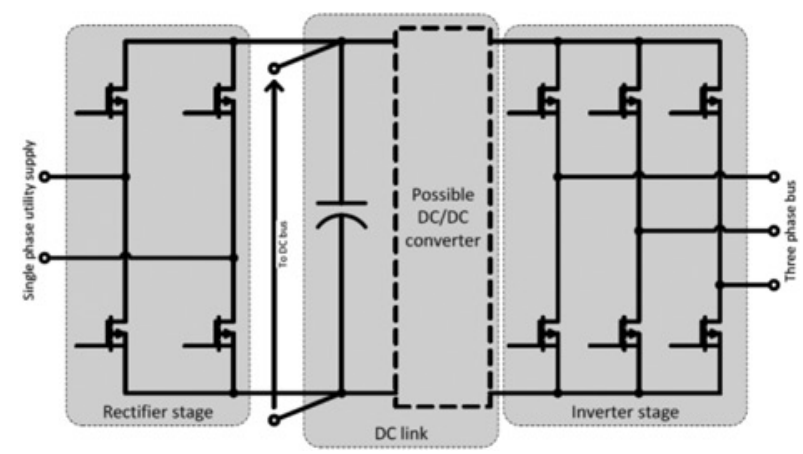

Fig. 1 Phase converter (source [2])

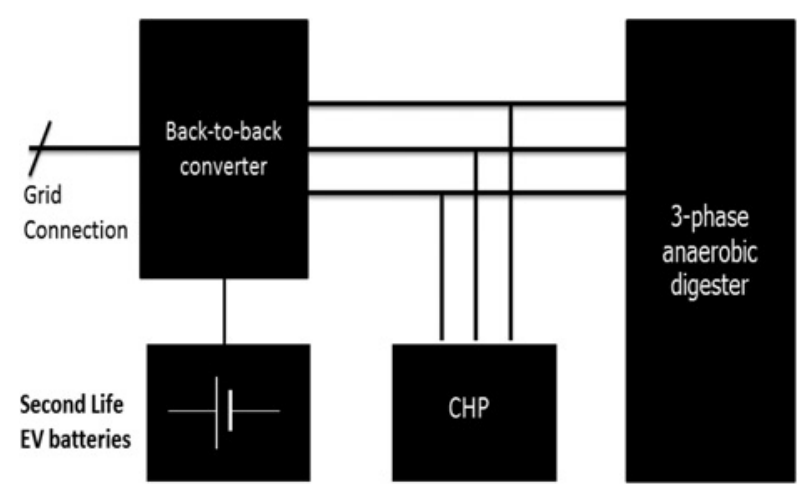

Fig. 2 Connection schematic for farm application

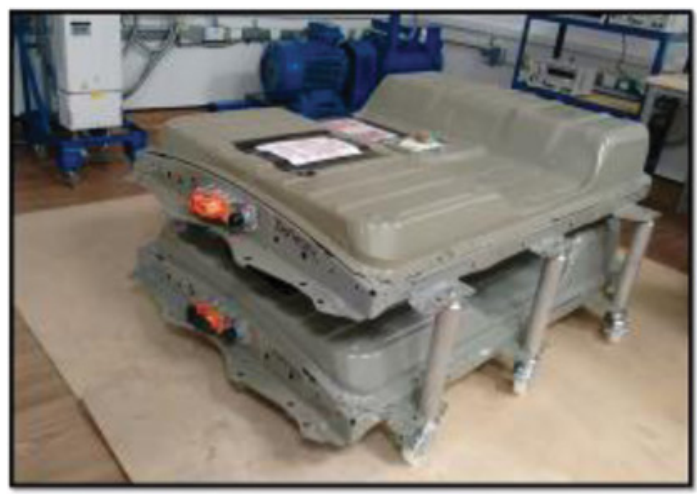

Fig. 3 Two used Nissan LEAF batteries

A DC/DC converter is indicated as a possible requirement in order to stabilise the DC link as well as raise/lower the voltage as necessary (Fig. 1). However, in order to keep the costs down, a DC/DC converter was not installed between the DC link and the battery array. Instead the DC-link busbar voltage is controlled to change from its nominal value to allow the charging or discharging of the batteries at a range of currents. A control loop is used to adjust the DC-link busbar voltage set point to obtain the desired current flow into or out of the battery. This is achieved independently to the flow of current through the back-to-back converter.

\section{Development process}

Newcastle University's smart grid laboratory has been used to develop the hardware required for the application at Cockle Park Farm. A systematic design process has been followed in order to take the steps needed to integrate the system components required in the final application. These are prototype and laboratory scale hardware, described below.

\subsection{Prototype}

For rapid prototyping we have used a power conversion system supplied by Triphase of Leuven. The control system is implemented in Matlab/Simulink which provides flexible and adaptable platform for development. The Simulink model is compiled as $\mathrm{C}$ code and runs on a Linux Real-Time Target computer. A number of options are available for passing information into and out of the hardware as it is operating. The DC rating of the converter is $10 \mathrm{~kW}$.

\subsection{Laboratory scale hardware - Naverino}

A small-scale capacity converter ( $12 \mathrm{kVA}$ ) has been installed in the laboratory with the same control capability as the full scale $(100 \mathrm{kVA})$ converter. This has allowed for the testing of the safety and control systems required for the real-world application within the laboratory environment. The laboratory scale converter can also operate as a single-phase to three-back back-to-back converter, so can also be used as a soft-open point.

Operations in the laboratory testing are now described.

\section{Control sequence}

The batteries are interfaced to the grid supply through a DC/AC converter. To connect the batteries to the converter a number of steps are required in preparation before enabling (dis)charging.

If there is a difference between the converter's DC voltage and the voltage of the battery at the point they are electrically connected, an uncontrolled and potentially large current will flow. Even when the control systems report that the voltages are equalised, a difference will likely still be there, so a pre-charge resistance as shown in Fig. 4 is interposed between the converter and the battery. This serves to limit the current that flows between the systems. Once the system is under closed-loop current control, the main connection between the converter and the battery can be closed and the pre-charge resistance removed.

Coordination of the operation of the connection relays is located within the starting sequence in Fig. 5. The starting sequence begins by starting the battery management system (BMS) and checking that the values read over the CAN bus are all within the applicable limits. Failure of any one of several values will prevent the sequence from proceeding and flag a fault. If the values are all ok, the process described above is initiated and completes with the relay sequence.

Now a loop is entered in which the values read from the BMS are continuously checked while the DC-link voltage is raised to charge the batteries or lowered to discharge the batteries. Closed-loop control

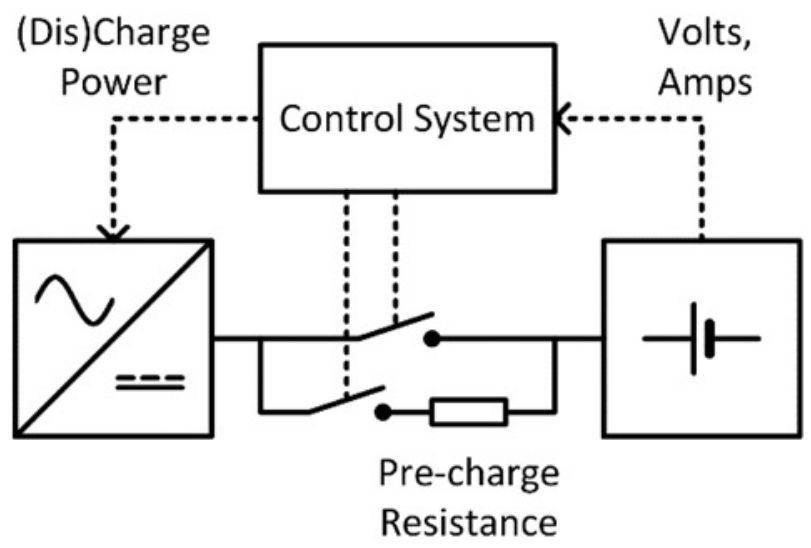

Fig. 4 Key system components: converter, relays, battery and control system 


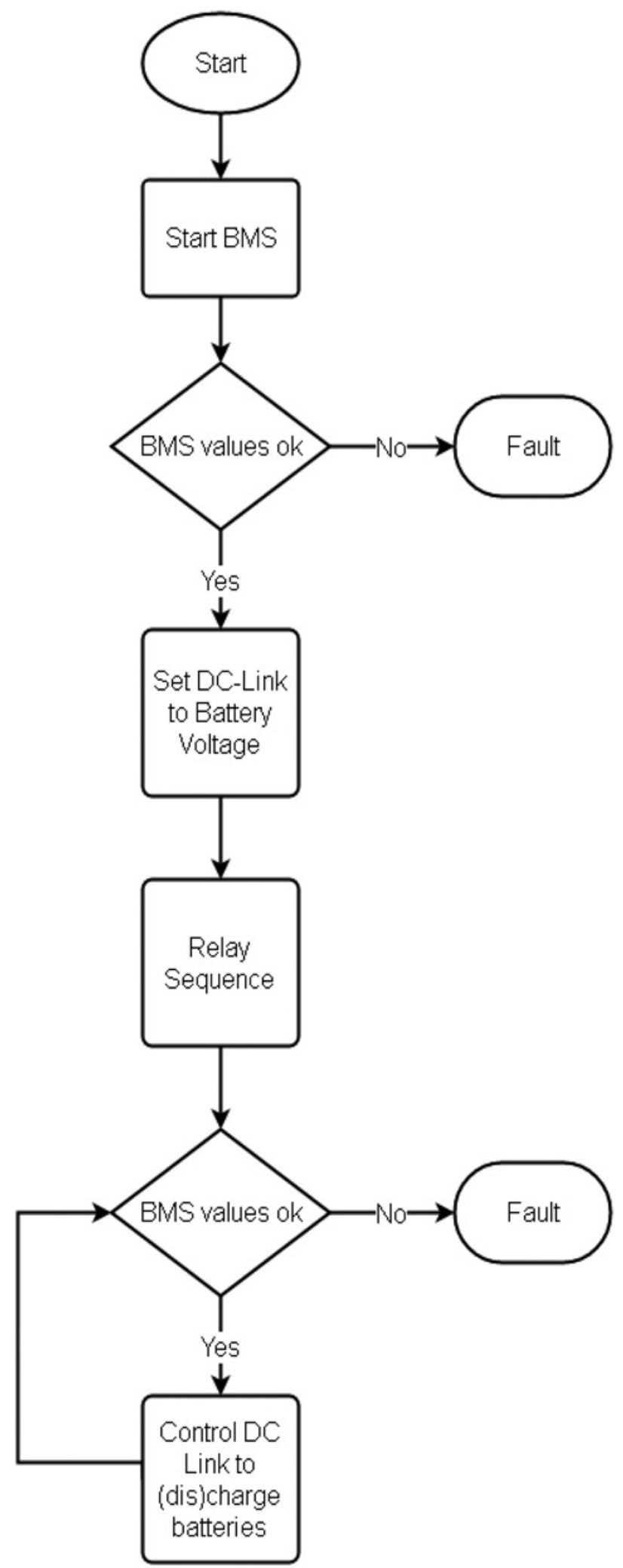

Fig. 5 Starting sequence to reach main control loop

adjusts the voltage set point to give the desired (dis)charge rate. Any anomalies in the BMS values result in the disconnection of the battery.

\section{Operation}

Once the electrical connection between the converter and battery has been established, current control can be used to regulate the exchange of power into and out of the battery. Fig. 6 shows a

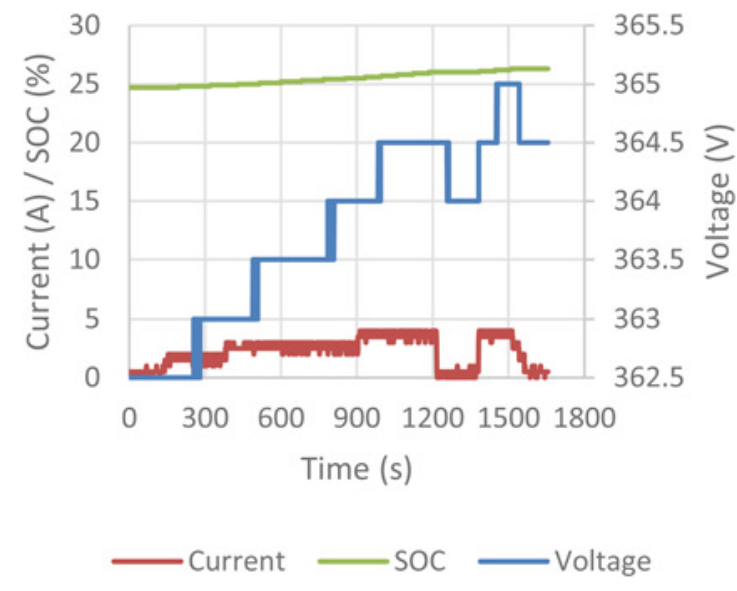

Fig. 6 Readings from BMS during charging operation

period of 30 min during which low current charging is carried out. While current is applied to the battery, the voltage gradually rises, with a fall in voltage when the current is turned off after $20 \mathrm{~min}$.

\section{Practical issues}

There needs to be an exchange of messages between the controller and battery sub-systems. This was implemented using user datagram protocol (UDP) communications between the controller and the dedicated UDP relay board actuating the battery contactors.

In designing the controller, provision was needed to continuously monitor the BMS values to be aware of warning signals and unsafe operating conditions. On detection of a failure condition the controller responds by disconnecting the battery from the converter.

As two batteries are needed to provide the voltage required for the back-to-back converter to operate on a 230Vac system, CAN messages need to be exchanged on two separate CAN buses. This was done by having two independent CAN channels on the control computer.

\section{$7 \quad$ DC link}

The $12 \mathrm{kVA}$ laboratory scale hardware and $100 \mathrm{kVA}$ application device are both controlled by sending a set point for the DC-link voltage over a UDP connection. This means that the controller that drives the converter can be changed without complication in accessing this set point.

\section{Discussion}

By procuring a full scale $100 \mathrm{kVA}$ converter and deploying it to serve a real-world application and also a laboratory scale $12 \mathrm{kVA}$ converter with similar characteristics it has been possible to develop and test control systems in a laboratory environment. Several difficulties have been overcome without the need for repeated visits to the site of the $100 \mathrm{kVA}$ converter.

The laboratory scale converter can be configured in several ways, so that as well as being able to perform tests that will be implemented on the larger system, alternative uses can also be investigated. This includes operation as a soft-open point within Newcastle University's Smart Grid Laboratory.

It is worth noting that data is collected from the battery in order assess capacity change which will be investigated in future work.

\section{Summary}

This paper has described the rationale for the installation of a $100 \mathrm{kVA}$ single- to three-phase converter at Newcastle 
University's Cockle Park Farm. This enables the operation of an anaerobic digestion plant which produces biogas to run a CHP system.

The converter system has a battery connected to the DC bus of the converter which can be charge and discharged by varying the DC bus set point. This has been prototyped in Newcastle University's smart grid laboratory and several challenges have been overcome on a smaller, laboratory scale, version of the $100 \mathrm{kVA}$ converter.

\section{Acknowledgments}

The authors gratefully acknowledge the contribution of Newcastle Institute for Sustainability and the support of Nissan for providing the used EV batteries.

\section{References}

1 Cipriano, E., Jacobina, C. B., da Silva, E. R. C., et al.: 'Single-phase to three-phase power converters: state of the art', IEEE Trans. Power Electron., 2012, 27, (5), pp. $2437-2452$

2 Sciberras, E., Widmer, J., Heslop, J.: 'Cockle park farm energy research impact study-facilitating rural energy for tomorrow', Newcastle University

3 'Anaerobic digestion the official information portal on anaerobic digestion'

4 Nykvist, B., Nilsson, M.: 'Rapidly falling costs of battery packs for electric vehicles', Nat. Clim. Change, 2015, 5, (4), pp. 329-332

5 Neubauer, J., Wood, E., Pesaran, A.: 'A second life for electric vehicle batteries: answering questions on battery degradation and value', SAE Int. J. Mater. Manuf., 2015, 8, (2), pp. 544-553, doi: 10.4271/2015-01-1306

6 Gladwin, D. T., Gould, C. R., Stone, D. A., et al.: 'Viability of second-life use of electric and hybridelectric vehicle battery packs'. IECON 2013 - 39th Annual Conf. IEEE Industrial Electronics Society, Vienna, Austria, 2013, pp. 1922 1927 